\title{
Weitergabe von Rabatten auf Rechnungen
}

\author{
Patrick Müller \\ Abteilungsleiter, Abteilung Ambulante Versorgung und Tarife, FMH
}

\begin{abstract}
Leistungserbringer müssen dem Schuldner einer Vergütung direkte oder indirekte Vergünstigungen weitergeben. Gesetz und Verordnung geben allerdings nicht vor, in welcher Form Preisrabatte auf Rechnungen ausgewiesen werden müssen. Die FMH hat deshalb eine Empfehlung erstellt, wie sich aus ihrer Sicht gewährte Rabatte transparent und gesetzeskonform auf Rechnungen ausweisen und weitergeben lassen.
\end{abstract}

Durch die Inkraftsetzung der neuen Art. 55 und 56 des Heilmittelgesetzes (HMG) ist seit dem 1. Januar 2020 das Interesse erneut und verstärkt auf die Problematik der «Weitergabepflicht von Rabatten und Vergünstigungen» gelenkt worden, welche seit 1996 grundsätzlich in Art. 56 Abs. 3 Krankenversicherungsgesetz (KVG) geregelt ist. Das KVG hält als Grundsatz die Pflicht zur vollumfänglichen Weitergabe von erhaltenen Vergünstigungen ${ }^{1}$ fest. Der Leistungserbringer muss dem Schuldner der Vergütung von Arzneimitteln oder Medizinprodukten direkte oder indirekte Vergünstigungen wie Preisrabatte und Rückvergütungen weitergeben (Art. 56 Abs. 3 KVG), im Tiers garant an Patientinnen und Patienten, im Tiers payant an die Krankenversicherer. Leistungserbringer dürfen erhaltene Vergünstigungen nicht für sich selbst behalten (Vorteilsverbot/Integritätsgebot) bzw. sind verpflichtet, allfällige Rabatte auf Medikamente und Medizinprodukte weiterzugeben (Weitergabepflicht).

\section{Transparenz und Nachvollziehbarkeit}

Der Art. 56 Abs. 3bis KVG definiert unter Einhaltung von Vorgaben gewisse Ausnahmen von der vollumfänglichen Weitergabepflicht. Diese Ausnahmen verlangen jedoch eine vorgängige Vereinbarung zwischen Leistungserbringer und Versicherer und sind aktuell nur im Rahmen von Art. 56 Abs. 3 lit. b KVG möglich also in Verbindung mit dem Kauf und Verkauf von Arzneimitteln sowie Mitteln und Gegenständen. Zudem müssen diese Vereinbarungen die folgenden Bedingungen erfüllen: Erstens müssen Vergünstigungen mehrheitlich weitergegeben werden, d.h. zu mehr als
50\% und weniger als 100\%, zweitens dürfen nicht weitergegebene Vergünstigungen ausschliesslich die Behandlungsqualität verbessern. Die FMH hat mit der Einkaufsgemeinschaft HSK von Helsana, sanitas und KPT und der CSS-Krankenversicherung einen Rahmenvertrag erarbeitet, in welchem die wichtigsten Grundsätze für die Umsetzung der neuen Bestimmungen festgehalten sind.

Das Gesetz und die Verordnung geben nicht vor, in welcher konkreten Form Preisrabatte auf Rechnungen ausgewiesen werden müssen. Ebenso wenig existieren gemeinsamen Empfehlungen der Tarifpartner, wie die in der Praxis sehr unterschiedlich gestalteten wirtschaftlichen Vereinbarungen zwischen den Akteuren auf einer Rechnung abgebildet werden können. Eine Anforderung ist sicherlich, dass dies in einer transparenten und nachvollziehbaren Form geschieht.

Die FMH hat deshalb eine Umsetzungsempfehlung erstellt, wie aus ihrer Sicht gewährte Rabatte auf den Rechnungen transparent nach Art. 42 KVG ausgewiesen und dem Schuldner der Vergütung weitergegeben werden können. Zudem gibt die Umsetzungsempfehlung einen Einblick in die betroffenen Bereiche der Heilmittel und Medizinprodukte. Erhaltene Rabatte können in unterschiedlichen Formen auf der Rechnung an den Schuldner weitergegeben werden. Im Sinne der Transparenz, Nachvollziehbarkeit und besseren Verständlichkeit empfiehlt die FMH den Ärztinnen und Ärzten, erhaltene Rabatte auf der Rechnung sowohl im Tiers garant (Rechnungsstellung an Patientin/Patient) wie auch im Tiers payant (Rechnungsstellung direkt an die Versicherung) wie folgt auszuweisen: 


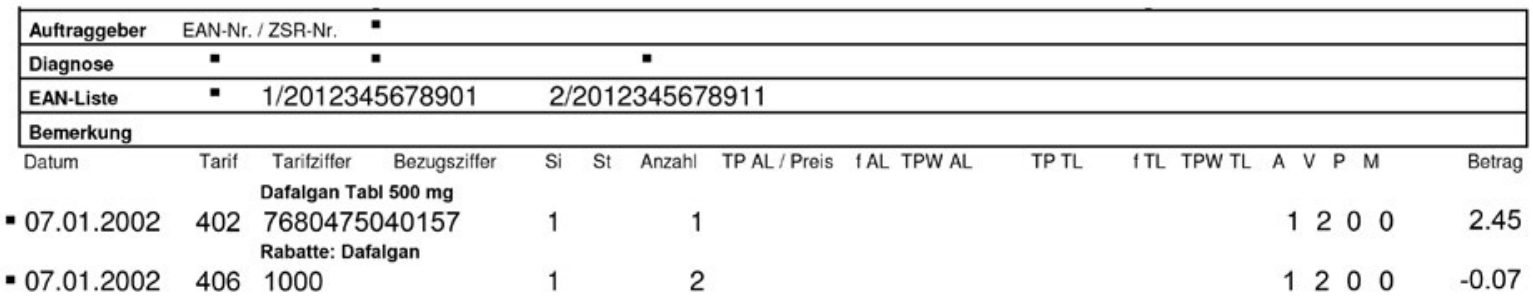

Abbildung 1: Rechnungsbeispiel eines Heilmittels mit gewährtem Preisrabatt von 5\% (CHF 0.07) auf den Fabrikabgabepreis (CHF 1.33); eigene Darstellung FMH.

\section{Umsetzungsempfehlung der FMH}

2 Gestützt auf Rahmenvertrag FMH/HSK-CSS «Qualitätsvereinbarung gemäss Artikel 56 Absatz 3bis KVG».
FMH

Abteilung Ambulante Versorgung und Tarife Baslerstrasse 47 CH-4600 Olten Tel. 0313591230 Fax 0313591238 tarife.ambulant[at]fmh.ch
Die FMH empfiehlt, bis spätestens im Jahr $2022^{2}$ für erhaltene Rabatte und Vergünstigungen eine zusätzliche separate Position über den Tarifcode 406 und die Tarifziffer 1000 "Rabatte» auf der Rechnung aufzuführen. Das Medizinprodukt oder Heilmittel soll mit dem ursprünglichen Betrag ohne Rabatt ausgewiesen werden (beispielsweise gemäss SL-Publikumspreis bei einem Heilmittel). Der effektiv gewährte Rabatt wird mit dem Frankenbetrag auf der Rechnung mit einer separaten Position ausgewiesen ohne weitere Angaben von gewährten Prozenten. Dabei kann der Positionstext der «Rabattposition» auf die spezifische Situation angepasst werden. Für jedes Produkt auf der Rechnung wird der Rabatt dabei separat ausgewiesen; die Tarifziffer 1000 ist abhängig von der Anzahl rabattierter Produkte also mehrmals anzuwenden.

In Abbildung 1 ist ein Heilmittel als Beispiel auf dem einheitlichen Rechnungsformular aufgeführt. Der gewährte Rabatt wird als Minusposition auf der Rechnung genannt. Dabei wird das Heilmittel im gezeigten Beispiel mit dem ordentlichen Publikumspreis der Spezialitätenliste SL verrechnet, und der auf dem Fabrikabgabepreis gewährte Preisrabatt wird mit der separaten Tarifposition 1000 «Rabatte» in Abzug gebracht.

\section{Vorteile der Umsetzungsempfehlung der FMH}

Die FMH hat versucht, diese Umsetzungsempfehlung mit den Krankenversicherungen zu vereinbaren. Diese bevorzugen jedoch eine Lösung über den Skalierungsfaktor (external factor). Die FMH lehnt diese Umset- zungsvariante jedoch klar ab und empfiehlt die Lösung über den Tarifcode 406 mit der Tarifziffer 1000 «Rabatte». Die Umsetzungsempfehlung der FMH ist aus vielen Gründen die bessere Variante. Sie ermöglicht eine transparente Darstellung auf der Rechnung, die für alle einfach nachvollziehbar ist - auch für die Patientinnen und Patienten.

Als Berufsverband der Schweizer Ärztinnen und Ärzte legt die FMH grossen Wert auf eine korrekte Umsetzung der Verordnung über die Integrität und Transparenz (VITH) und möchte Transparenz und Nachvollziehbarkeit für alle sicherstellen. Für diesen Zweck eignet sich eine spezifische Tarifposition optimal und ist ferner einfach monitorisierbar. Durch die separate Ausweisung von Originalpreisen und Rabatten ist die Höhe von Vergünstigungen auf einen Blick ersichtlich und verdeutlicht so, wie Leistungserbringer und Lieferanten zu Einsparungen im Gesundheitswesen beitragen. Diese Einsparungen können über die Tarifposition aber nicht nur auf einer einzelnen Rechnung transparent dargestellt werden, sondern lassen sich beispielsweise auch für die gesamte ambulant tätige Ärzteschaft aggregieren und auswerten.

\section{Weitere Informationen}

Bei Fragen zur neuen Umsetzungsempfehlung der FMH können Sie gerne die Abteilung Rechtsdienst (lex[at]fmh.ch) oder die Abteilung Ambulante Versorgung und Tarife (tarife.ambulant[at]fmh.ch) kontaktieren. Zudem finden Sie auf der Website der FMH unter www.fmh.ch $>$ Dienstleistungen $>$ Recht $>$ Verordnung über die Integrität und Transparenz VITH weitere Informationen. Die Umsetzungsempfehlung ist ebenfalls auf der Website der FMH einzusehen. 